\title{
A New Version of the SOLAR-ISS Spectrum Covering the 165-3000 nm Spectral Region
}

\author{
M. Meftah ${ }^{1}$. L. Damé ${ }^{1}$. D. Bolsée ${ }^{2}$. \\ N. Pereira $^{2}$ - M. Snow ${ }^{3}$. M. Weber ${ }^{4}$. \\ K. Bramstedt ${ }^{4}$ - T. Hilbig ${ }^{4}$ - G. Cessateur ${ }^{2}$. \\ M.-Y. Boudjella ${ }^{1,5}$ - M. Marchand ${ }^{1}$. \\ F. Lefèvre ${ }^{1}$ - R. Thiéblemont ${ }^{1}$. \\ A. Sarkissian ${ }^{1}$. A. Hauchecorne ${ }^{1}$. \\ P. Keckhut ${ }^{1}$. S. Bekki ${ }^{1}$.
}

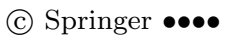

\begin{abstract}
The accurate measurement of the solar spectrum at the top of the atmosphere and its variability are fundamental inputs for solar physics (Sun modeling), terrestrial atmospheric photochemistry and Earth's climate (climate's modeling). These inputs were the prime objective set in 1996 for the SOLAR mission. The SOLAR package represents a set of three solar instruments measuring the total and spectral absolute irradiance from $16 \mathrm{~nm}$ to $3088 \mathrm{~nm}$. SOLAR was launched with the European Columbus space laboratory in February 2008 aboard the NASA Space Shuttle Atlantis. SOLAR on the International Space Station (ISS) tracked the Sun until it was decommissioned in February 2017. The SOLar SPECtrum (SOLSPEC) instrument of the SOLAR payload allowed the measurement of solar spectra in the $165-3000 \mathrm{~nm}$ wavelength range for almost a decade. Until the end of its mission, SOLAR/SOLSPEC was pushed to its limits to test how it was affected by space environmental effects (external thermal factors) and to better calibrate the space-based spectrometer. To that end, a new solar reference spectrum (SOLAR-ISS - V1.1) representative of the 2008 solar minimum was obtained from the measurements made by the SOLAR/SOLSPEC instrument and its calibrations. The main purpose of this article is to improve the SOLAR-ISS reference spectrum (between 165 and $180 \mathrm{~nm}$ in the far ultraviolet, between 216.9 and $226.8 \mathrm{~nm}$ in the middle ultraviolet, and
\end{abstract}

\footnotetext{
1 LATMOS, UVSQ, Université Paris Saclay, Sorbonne Université, CNRS, INSU, IPSL, 11 Boulevard d'Alembert, 78280 Guyancourt, France

Email: Mustapha.Meftah@latmos.ipsl.fr

2 Belgian Institute for Space Aeronomy (BIRA-IASB), Ringlaan 3, B-1180 Brussels, Belgium

${ }^{3}$ Laboratory for Atmospheric and Space Physics, University of Colorado, 3665 Discovery Drive, Boulder, Colorado, USA

${ }^{4}$ Institute of Environmental Physics, University of Bremen, 28359 Bremen, Germany

5 LAAR, USTO-MB, Bir El Djir, 31000 Oran, Algeria
} 
between 2400 and $3000 \mathrm{~nm}$ in the near-infrared). SOLAR-ISS has a resolution better than $0.1 \mathrm{~nm}$ between 165 and $1000 \mathrm{~nm}$, and $1 \mathrm{~nm}$ in the $1000-3000 \mathrm{~nm}$ wavelength range. Finally, a first comparison is made between the new SOLARISS spectrum (V2.0) and the Total and Spectral solar Irradiance Sensor (TSIS-1) spectrum obtained from its first observations from the ISS. Indeed, the launch of TSIS in December 2017 provides a new light on the absolute determination of the solar spectrum and especially in the infrared region of the spectrum.

Keywords: Solar Irradiance; Solar Cycle; SOLAR-ISS (V2.0); TSIS.

\section{Introduction}

For around 9 years since April 5, 2008 and until February 15, 2017, the SOLar SPECtrum (SOLSPEC) instrument of the SOLAR facility on the International Space Station (ISS) performed accurate measurements of Solar Spectral Irradiance (SSI) from the far ultraviolet to the mid-infrared $(165-3000 \mathrm{~nm})$. The SOLAR/SOLSPEC spectro-radiometer (Thuillier et al., 2009; Bolsée, 2012) was designed to work for only 18 months, but was switched off after almost a decade. This performance over time is linked to the fact that the SOLAR/SOLSPEC instrument was improved with the experience and heritage gained from previous space-based missions (Spacelab-1 in 1983, ATLAS-1 in 1992, EURECA between 1992 and 1993, ATLAS-2 in 1993, and ATLAS-3 in November 1994). Thus, the primary objective of the SOLAR mission was the measurement of the solar spectral irradiance with the highest possible accuracy, and its variability over time. Space-based SSI measurements are crucial for solar energy (Gueymard, 2018), space weather, and our understanding of the Sun-Earth connection as explained by Snow et al. (2018). Accurate measurements are necessary to better understand the impact of solar variability on climate (via Earth's atmospheric photochemistry), noticeably through the "top-down" mechanism amplifying ultraviolet solar forcing effects on the climate (UV affects stratospheric dynamics and temperatures, altering interplanetary waves and weather patterns both poleward and downward to the lower stratosphere and troposphere regions).

Our objective is to produce a spectral irradiance dataset based on recent SOLAR/SOLSPEC measurements obtained during the 2008-2017 period. The SOLAR/SOLSPEC measurements, unique by their large spectral coverage and long time range, are of primary importance. During this period of observation, the only alternative solar spectral irradiance measurements are those from the Solar Radiation and Climate Experiment (SORCE) satellite in the $0.1-2412 \mathrm{~nm}$ wavelength range (Rottman, 2005) and from the Aura Ozone Monitoring Instrument (OMI) in the 265-500 nm wavelength range (Marchenko, DeLand, and Lean, 2016). Thus, it is highly recommended to have several SSI datasets from different space-based instruments to verify the accuracy and the confidence of the calibrated solar measurements. The main goal of our current studies is to deliver the evolution of the SOLAR/SOLSPEC spectral irradiance during Cycle 24 thanks to revised engineering corrections, improved calibrations, and advanced procedures to account for thermal and aging corrections 
of SOLAR/SOLSPEC data. A preliminary version of the partial dataset has already been described in the literature (Meftah et al., 2018), presenting a new reference solar spectrum for the 2008 solar minimum (SOLAR-ISS - Version 1.1). Above $1500 \mathrm{~nm}$, SOLAR-ISS shows significant differences with other reference spectra such as the ATLAS $3(0.5-2397.51 \mathrm{~nm})$ reference solar spectrum (Thuillier et al., 2003) and the Solar Irradiance Reference Spectra (Woods et al., 2009) for the 2008 Whole Heliosphere Interval (WHI 2008; $0.1-2400 \mathrm{~nm}$ ). Recent studies (Figure 1) are consistent with the SOLAR-ISS spectrum values above $1500 \mathrm{~nm}$. Indeed, the SOLAR-ISS reference spectrum agrees well with the SCanning Imaging Absorption spectroMeter for Atmospheric CHartographY (SCIAMACHY; 240-2400 nm) solar spectrum (Hilbig et al., 2018) and recent ground-based measurements obtained during the PYR-ILIOS SSI near infrared (NIR) ground-based campaign made in Mauna Loa Observatory in July 2016 (Pereira et al., 2018). Moreover, the measurement campaign of the Continuum Absorption in the Visible and Infrared and its Atmospheric Relevance (CAVIAR 2; $1400-2500 \mathrm{~nm}$ ) project (Elsey et al., 2017) shows a good agreement with the SOLAR-ISS spectrum, but significant differences from the ATLAS 3 solar spectrum ( $\sim 7 \%)$. Menang (2018) specifies that the rigorous analysis methods and updated calibrations employed to derive CAVIAR 2 and SOLAR-ISS SSI are strong indicators that these two spectra may be more reliable.

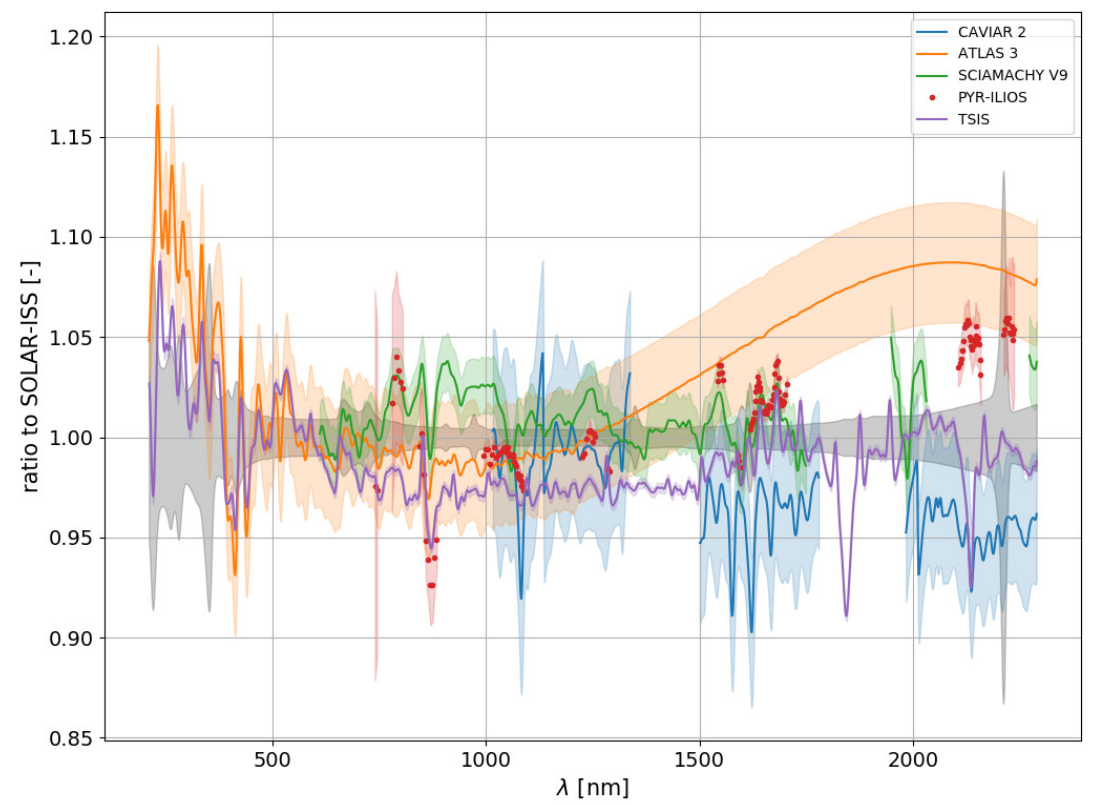

Figure 1. Ratio to SOLAR-ISS (V1.1) for CAVIAR 2, ATLAS 3, SCIAMACHY (V9), PYR-ILIOS, and TSIS. Above $1500 \mathrm{~nm}$, all recent results agree well with the SOLAR-ISS reference spectrum.

With the launch of the Total and Spectral solar Irradiance Sensor (TSIS-1) mission in December 2017, new solar spectral irradiance observations (200$2400 \mathrm{~nm}$ ) are available (http://lasp.colorado.edu/data/tsis) since March 2018 af- 
ter two months of instrument commissioning (Richard et al., 2016; Richard et al., 2018). These measurements are essential to confirm or not the SOLAR-ISS spectrum, and particularly above $1500 \mathrm{~nm}$.

The first objective of this article is to provide an improvement of the SOLARISS reference spectrum (from V1.1 to V2.0). There are modifications in the UV part of the solar spectrum in the $165-180 \mathrm{~nm}$ spectral region (fit of the SOLAR-ISS spectrum with SOlar Stellar Irradiance Comparison Experiment (SOLSTICE) observations in April 2008) and in the 216.9-226.8 nm spectral region (modification of the instrument's spectral irradiance response). There are also modifications in the NIR between 2400 and $3000 \mathrm{~nm}$ (suppression of a few solar lines using another solar model for the high resolution that match better with solar pseudo-transmissions based on observations). The second objective is to make a comparison between SOLAR-ISS (V2.0) and recent SSI observations obtained with TSIS-1. Then, in a part of the UV spectrum $(165-320 \mathrm{~nm})$, a comparison will be done between SOLAR-ISS and the observations obtained in April 2008 with SOLSTICE (120-320 nm) onboard the SORCE satellite.

\section{Data \& Methods used to obtain the SOLAR-ISS Spectrum}

\subsection{SOLAR/SOLSPEC Data and General Method}

SOLAR/SOLSPEC measured the solar spectrum in the $165-3000 \mathrm{~nm}$ spectral region. The spectro-radiometer consists of 3 separated double-monochromators that use concave holographic gratings to cover "UV" (165-371 nm), "VIS" (285$908 \mathrm{~nm})$, and "IR" (646-3088 nm) spectral ranges. For each SOLAR/SOLSPEC double-monochromator (Bolsée, 2012), we use an instrumental equation (Meftah et al., 2018) linked with engineering corrections and improved calibrations. Theses three instrumental corrections allow to derive the solar spectral irradiance from SOLAR/SOLSPEC raw data. In addition, we have an excellent knowledge of the bandpass (spectral resolution commonly called the slit function) for each SOLAR/SOLSPEC double-monochromator. Therefore, we can obtain a higher resolution spectrum (SOLAR-ISS) by using the Equation 1 as explained in more details in Meftah et al. (2018).

$$
\operatorname{SOLAR}-\operatorname{ISS}(\lambda)=\operatorname{SSI}_{\mathrm{HR}}(\lambda) \times \frac{\operatorname{SSI}_{\operatorname{SOLSPEC}}(\lambda)}{\operatorname{SSI}_{\mathrm{HR}}(\lambda) \otimes \operatorname{SF}_{\operatorname{SOLPPC}}(\lambda)}
$$

where $\operatorname{SSI}_{\mathrm{HR}}(\lambda)$ is the high-resolution solar spectrum (Kurucz and Bell, 1995), $\operatorname{SSI}_{\operatorname{SOLSPEC}}(\lambda)$ is the SOLAR/SOLSPEC spectrum obtained from its three double-monochromators, $\operatorname{SF}_{\operatorname{SOLSPEC}}(\lambda)$ represents the slit functions of the SOLAR/SOLSPEC instrument, and $\otimes$ denotes the convolution symbol.

\subsection{SOLAR/SOLSPEC calibrations and Uncertainty Budget}

Before launch of the SOLAR package in February 2008, several characteristics of the SOLAR/SOLSPEC instrument (Thuillier et al., 2009; Bolsée, 2012) were 
accurately measured for each spectrometer such as absolute response, linearity, slit functions (Meftah et al., 2018) \& bandpass (full width at half maximum of the spectral response), dispersion law, spectral scanning stability, instrument scattered light, field of view, etc.

The SOLAR/SOLSPEC absolute response calibration is very important for the absolute determination of the 'true' solar spectrum. Between 165 and below $200 \mathrm{~nm}$, this calibration was performed by the BIRA-IASB/LATMOS team in 2007, using a thermal vacuum chamber and deuterium lamps (Cathodeon Nos. V0132 and BR066) calibrated under vacuum in radiant intensity at PhysikalischTechnische Bundesanstalt (PTB, BESSY II facility, Berlin, Germany) (Bolsée et al., 2017). For wavelengths between 200 and $3000 \mathrm{~nm}$, the absolute response calibration of the SOLAR/SOLSPEC instrument was carried out at PTB using the BB3200pg blackbody source, which is a primary standard for the realization and dissemination of spectral irradiance. The BB3200pg blackbody provides a very stable reference source of known spectral radiance using the Planck's law. The uncertainty on the SOLAR/SOLSPEC absolute response acts as a source of systematic errors in space and represents the dominant and irreducible contribution in the uncertainty budget of the SOLAR/SOLSPEC SSI measurement in space (Bolsée et al., 2017). We also developed a range of processing and correction methods (thermal behavior effects of the instrument), which are described in detail by Bolsée et al. (2017) and Meftah et al. (2017). These corrections have also introduced contributions in the uncertainty budget of the SOLAR/SOLSPEC SSI measurement.

The SOLAR-ISS spectrum has a mean absolute uncertainty of $\sim 1.26 \%(\sim 16.9$ $\mathrm{W} \mathrm{m}^{-2}$ ) at $1 \sigma$ in the $165-3000 \mathrm{~nm}$ spectral region (Meftah et al., 2018). The integral of SOLAR-ISS in the $165-3000 \mathrm{~nm}$ range is between $1344-1345 \mathrm{~W} \mathrm{~m}^{-2}$. This value assumes the SOLAR-ISS total solar irradiance (TSI) of $\sim 1372.5$ $\mathrm{W} \mathrm{m}^{-2}$ and estimates the irradiance in the $3-100 \mu \mathrm{m}$ wavelength range at a value of $\sim 28 \mathrm{~W} \mathrm{~m}^{-2}$ using a model (spectrum of a blackbody at $5772 \mathrm{~K}$, Shapiro et al. (2010) code for solar irradiance modeling (COSI), Tagirov, Shapiro, and Schmutz (2017) non-local thermodynamic equilibrium spectral synthesis code (NESSY), Fontenla and Landi (2018) physical modeling of solar spectral irradiance, etc.). It has been established that the TSI value is close to $1361 \mathrm{Wm}^{-2}$ (Kopp and Lean, 2011; Schmutz et al., 2013; Meftah et al., 2014) and represents a TSI community-consensus value (Kopp et al., 2018). Thus, the difference between a TSI of $1361 \mathrm{Wm}^{-2}$ representative of a solar minimum and the SOLARISS TSI is of the order of $11.5 \mathrm{~W} \mathrm{~m}^{-2}$. This difference is consistent with the mean absolute uncertainty of the SOLAR-ISS spectrum. A uniform reduction of $\sim 0.84 \%$ applied to the SOLAR-ISS spectrum in the $165-3000 \mathrm{~nm}$ range would match with a TSI of $1361 \mathrm{Wm}^{-2}$ (outcome of the International Astronomical Union (IAU) 2015 Resolution B3). However, the SOLAR/SOLSPEC measurements uncertainties and the possible errors on the absolute determination of SSI are not the same in the $165-3000 \mathrm{~nm}$ range. Therefore, we do not recommend that the irradiance values are properly scaled so as to integrate to $1361 \mathrm{Wm}^{-2}$. 


\section{SOLAR-ISS (LR) improvements}

The SOLAR-ISS spectrum is obtained from the SOLAR/SOLSPEC observations, from which the $\operatorname{SSI}_{\mathrm{SOLSPEC}}(\lambda)$ spectrum at low resolution (SOLAR-ISS $(\mathrm{LR})$ ) is derived, as explained in Section 2.1. An improvement of the SOLARISS spectrum requires an improvement of SOLAR-ISS (LR) associated with a very accurate knowledge of the slit functions $\left(\operatorname{SF}_{\operatorname{SOLSPEC}}(\lambda)\right)$ of the SOLAR/SOLSPEC instrument considered to be fully acquired (Meftah et al., 2018). Solar cycle 24 provides a possibility of inter-comparison of solar spectral irradiance for SOLAR/SOLSPEC and SORCE/SOLSTICE instruments, which allows us to consider solar spectrum improvements.

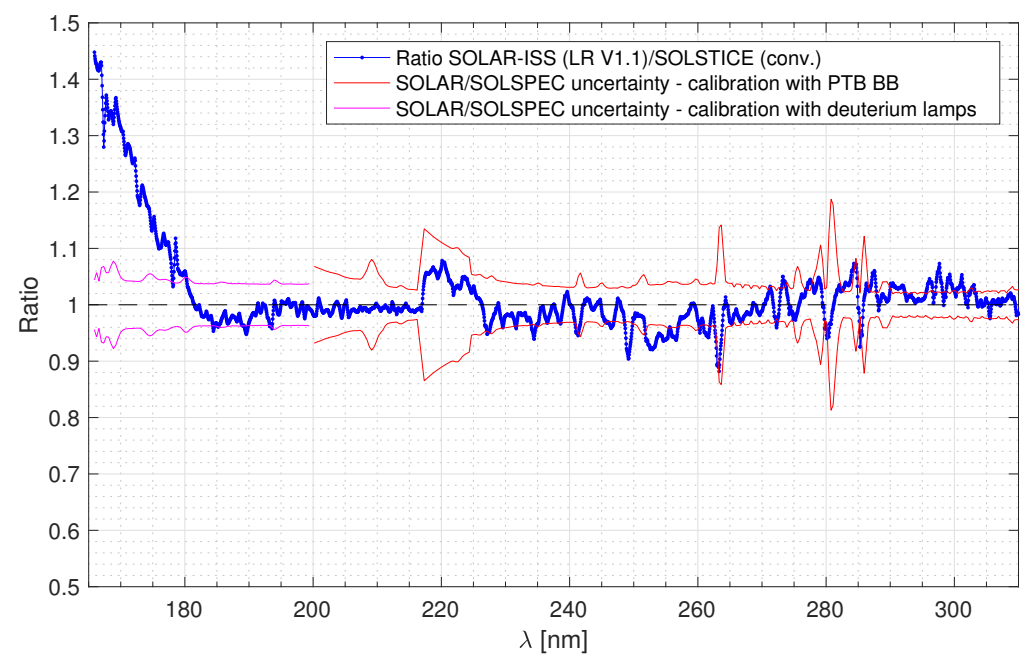

Figure 2. SOLAR-ISS (LR)/SOLSTICE ratio during the same period of observation in April 2008. The magenta curve highlights the absolute uncertainty $(1 \sigma)$ of the SOLAR-ISS spectrum in the $165-200 \mathrm{~nm}$ range (calibration with the deuterium lamps calibrated by PTB in vacuum). The red curve represents the absolute uncertainty $(1 \sigma)$ of the SOLAR-ISS spectrum in the $200-310 \mathrm{~nm}$ range (calibration with the PTB blackbody (BB)). The SOLAR/SOLSPEC slit functions are used for convolving the SORCE/SOLSTICE spectrum at the SOLAR/SOLSPEC spectral resolution.

Figure 2 shows the ratio between SOLAR-ISS (LR V1.1) and the last version available of the SORCE/SOLSTICE solar spectrum representative of April 2008 observations. The comparison between SOLAR/ISS and SORCE/SOLSTICE spectra highlights a spectral region $(165-180 \mathrm{~nm})$ with a significant difference. This comparison shows relative uncertainties against the SORCE/SOLSTICE spectrum of nearly $45 \%$ differences in the shortest wavelength region $(165 \mathrm{~nm})$. It should be noted that SORCE/SOLSTICE measurements transition from the MUV detector $(170-320 \mathrm{~nm})$ for wavelengths longward of $180 \mathrm{~nm}$ to the FUV detector $(115-180 \mathrm{~nm})$ for wavelengths shortward of $180 \mathrm{~nm}$. However, other publications using these data do not show evidence of any errors that affect only FUV measurements.

Otherwise, the SOLAR-ISS (LR)/SOLSTICE ratio also highlights a strong irregularities in the Herzberg continuum $(216.9-226.8 \mathrm{~nm})$, which is also problematic. 
Therefore, the SOLAR-ISS (LR V1.1) spectrum (Figure 3) is questionable in these two spectral regions.
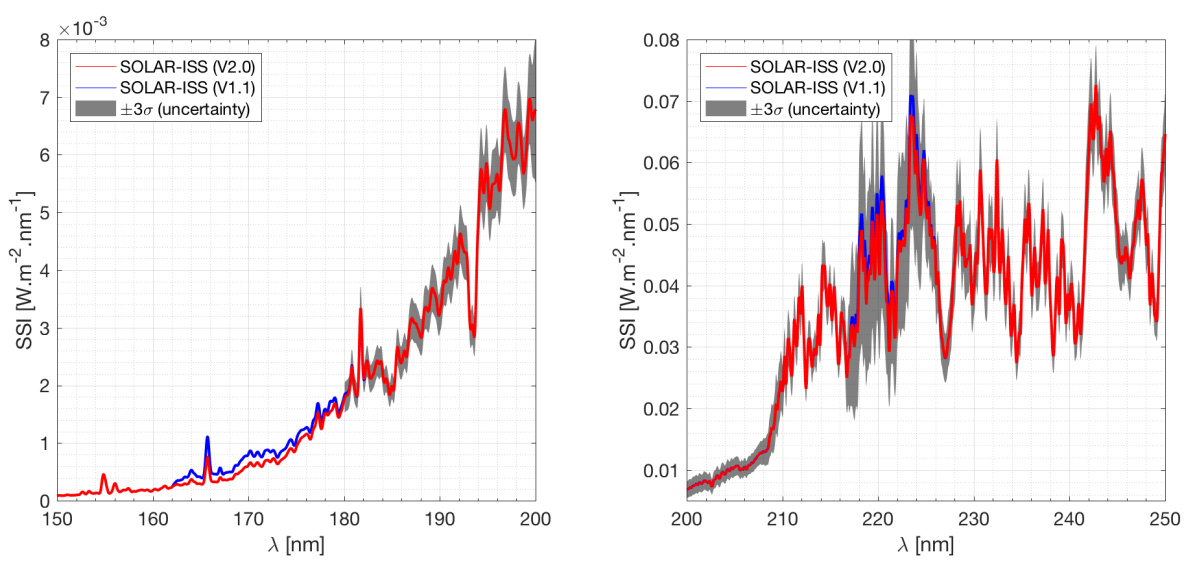

Figure 3. Left: Solar spectral irradiance of the two versions of the SOLAR-ISS spectrum in the $165-200 \mathrm{~nm}$ spectral region. Right: Comparison between the two versions of the SOLAR-ISS spectrum in the $200-250 \mathrm{~nm}$ spectral region.

\subsection{Improvements in the $165-180 \mathrm{~nm}$ spectral region}

Between 165 and $180 \mathrm{~nm}$, the SOLAR-ISS (LR V1.1)/SOLSTICE ratio presents significant differences with values close to 1.45 at $165 \mathrm{~nm}$. Between 165 and $180 \mathrm{~nm}$, the SOLAR/SOLSPEC instrument was not calibrated with the PTB blackbody (see Section 2.2), whose is tied to radiance standards. Indeed, the SOLAR/SOLSPEC calibration was done in Laboratory because the PTB blackbody is not usable below $200 \mathrm{~nm}$. A deuterium lamp calibrated in PTB and SOLAR/SOLSPEC were set in a vacuum chamber for doing this calibration. The problem of this calibration is related to the fact that the distance Instrument/Lamp was very short given the dimensions of the vacuum chamber. The terms of use of the deuterium lamp (V0132, Cathodeon Ltd, United Kingdom) near SOLAR/SOLSPEC may have been inappropriate (scattered light effect). A contribution could come from a reflection of the emerging beam of this lamp $\left(45^{\circ}\right.$ of divergence) on the walls of the instrument solar internal deflector (length of $90 \mathrm{~mm}$ ), which is located between the main shutter and the UV pre-slit. Due to this effect, measurements were made between 166 and $245 \mathrm{~nm}$ to ensure recovery with the response curve obtained during the PTB blackbody calibration. Therefore, the vacuum response curve established with the deuterium lamp was corrected by a multiplicative factor equal to $1 / 0.852$ given by the $200-245 \mathrm{~nm}$ overlapping region of the two calibrations (PTB blackbody and laboratory calibration in vacuum). This correction for the $165-200 \mathrm{~nm}$ absolute response is questionable and particularly for wavelengths which are far away from $200 \mathrm{~nm}$. Consequently, it is realistic to envisage that a difference related to a spectral dependence was not taken into account during the SOLAR/SOLSPEC calibration in vacuum chamber for wavelengths below $200 \mathrm{~nm}$. Moreover, additional sources of 
errors may also have been omitted in the methodology used for obtaining the SOLAR/SOLSPEC spectrum in the "UV" range (Meftah et al., 2016). Therefore, it seems reasonable to fit the SOLAR-ISS spectrum with SORCE/SOLSTICE for wavelengths below $180 \mathrm{~nm}$ because this is the only source of measurement available at the same date of observation. This adjustment is justified if we consider that the measurement uncertainty of SORCE/SOLSTICE is reliable, and that no other additional errors could be accounted for SORCE/SOLSTICE wavelengths between 165 and $180 \mathrm{~nm}$. This is the case because the SORCE/SOLSTICE instrument was designed to have an absolute accuracy of 5\% (in the entire wavelength range) with a relative accuracy of $0.5 \%$ per year to determine SSI during a 5-year nominal mission (McClintock, Rottman, and Woods, 2005). Snow, McClintock, and Woods (2010) show comparisons between SORCE/SOLSTICE and Upper Atmosphere Research Satellite (UARS) SOLSTICE data that help establish the accuracy of the SORCE/SOLSTICE measurements (the degradation correction for both SOLSTICE instruments is on the order of $0.5 \%$ per year). For the SORCE/SOLSTICE far UV channel (data access: http://lasp. colorado.edu/home/sorce/data/), the uncertainties are a combination of instrumental and statistical factors, as well as solar variability. In April 2008, the SSI SORCE/SOLSTICE absolute uncertainties are close to $3.3 \%$ at $1 \sigma$ in the $165-180 \mathrm{~nm}$ spectral region. As shown in Figure 2, measurements made by the SOLAR/SOLSPEC instrument are outside the uncertainty margins of the SORCE/SOLSTICE instrument in the $165-180 \mathrm{~nm}$.

Then, an improvement in UV seems necessary to consolidate SOLARISS for wavelengths between 165 and $180 \mathrm{~nm}$. Figure 3 (Left) shows the difference between the two versions of the SOLAR-ISS spectrum in the $165-180 \mathrm{~nm}$ spectral region. For the new version of the SOLARISS spectrum (V2.0), no uncertainty is associated in the $165-180 \mathrm{~nm}$ spectral region since we fit our data with SORCE/SOLSTICE. A future work (SOLAR/SOLSPEC UV spectrum, calibration, etc.) is required to understand the differences observed between SOLAR-ISS and SORCE/SOLSTICE in this spectral region (Figure 2).

\subsection{Improvements in the $216.9-226.8 \mathrm{~nm}$ spectral region}

Beyond $200 \mathrm{~nm}$ and up to $320 \mathrm{~nm}$, the SOLAR-ISS/SOLSTICE ratio remains in the margins of uncertainty both of SOLAR-ISS and the SORCE/SOLSTICE spectrum (Figure 2). However, irregularities between the SOLAR-ISS spectrum and the SORCE/SOLSTICE spectrum are observed in Figure 2, particularly in the $216.9-226.8 \mathrm{~nm}$ range.

The SOLAR-ISS/SOLSTICE ratio irregularity starting at $216.9 \mathrm{~nm}$ is related to the SOLAR/SOLSPEC calibration method. From $200 \mathrm{~nm}$, the absolute response calibration of the SOLAR/SOLSPEC instrument was carried out with the PTB blackbody and reinforced by the use of the D2 EF159 deuterium lamp (calibrated itself with the PTB blackbody). For the PTB calibration between 200 and $216.6 \mathrm{~nm}$, no attenuation optical filter was used between the exit slit and the SOLAR/SOLSPEC "UV" photomultiplier detector. From this calibration, the $\mathrm{R} 1$ response curve was obtained (Figure 4). For the $216.9-320 \mathrm{~nm}$ range 
calibration, a SOLAR/SOLSPEC actuator allowed us to add an attenuation optical filter between the exit slit and the "UV" photomultiplier detector. The purpose of this attenuation optical filter was to limit the number of counts per second on the SOLAR/SOLSPEC photomultiplier; it was requested to not exceed $10^{5}$ counts per second during a solar measurement to limit the effects of the photomultiplier non-linearity (LATMOS heritage). From the calibration with the attenuation optical filter, the R2 response curve was obtained (Figure 4). The two SOLAR/SOLSPEC response curves (R1 \& R2) highlight the transition region between 216.6 and $216.9 \mathrm{~nm}$. Close to $216.9 \mathrm{~nm}$, the signalto-noise ratio is weak due to the SOLAR/SOLSPEC attenuation optical filter, which results in increasing the measurement uncertainty in the $216.9-226.8 \mathrm{~nm}$ spectral region (Figure 2). The irregularity in the $216.9-226.8 \mathrm{~nm}$ range is linked with the use of the SOLAR/SOLSPEC attenuation optical filter when switching to $216.9 \mathrm{~nm}$. Therefore, a SOLAR-ISS improvement in the $216.9-226.8 \mathrm{~nm}$ range is required by fitting SOLAR-ISS with SORCE/SOLSTICE data during the same period of observation in April 2008. This approach seems reasonable given the good agreement between datasets (differences less than $4 \%$, no spectral dependence) seen in Figure 2 for the rest of the $180-250 \mathrm{~nm}$ region. The TSIS observations bring a new light to confirm that the signature of the SOLARISS/TSIS ratio in the $216.9-226.8 \mathrm{~nm}$ range is similar to that obtained with the SOLAR-ISS/SOLSTICE ratio.

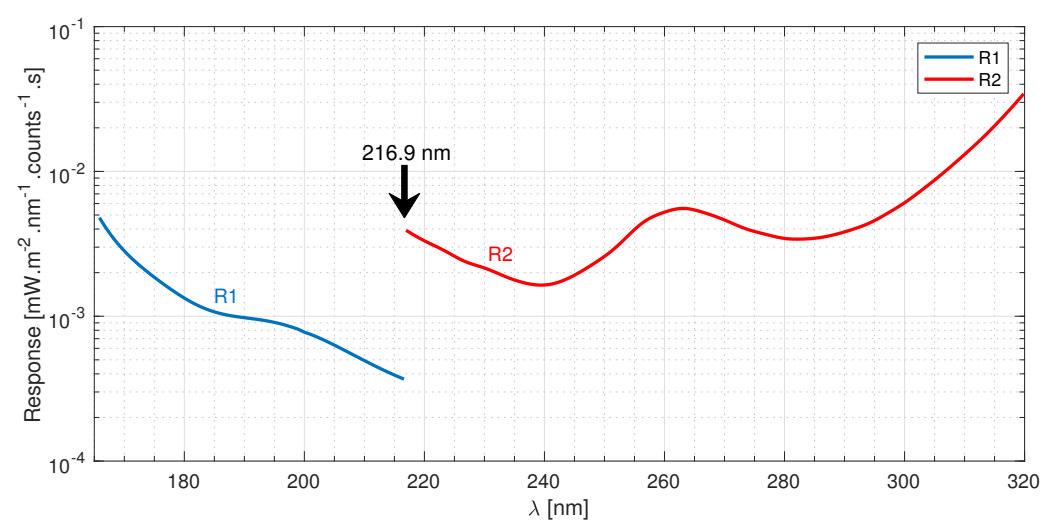

Figure 4. The blue curve corresponds to the SOLAR/SOLSPEC absolute response (R1) in the $165-216.6 \mathrm{~nm}$ range. The red curve corresponds to the SOLAR/SOLSPEC absolute response (R2) in the $216.9-320 \mathrm{~nm}$ range.

The SOLAR-ISS improvement in the $216.9-226.8 \mathrm{~nm}$ is important because it corresponds to a spectral region that is included in the Herzberg continuum $(200-242 \mathrm{~nm})$. In this region, atmospheric absorption is relatively low and hence solar UV radiation penetrates deeply in the atmosphere, down to the lower stratosphere, where it photolysis molecular oxygen $\left(O_{2}\right)$ to produce ozone $\left(O_{3}\right)$. Absolute solar spectral irradiance and variability in the Herzberg continuum are necessary to better understand the stratospheric ozone response to solar UV irradiance changes (Brasseur and Solomon, 2005). Other analysis related 
to the study of fundamental physical and chemical processes governing the terrestrial and planetary atmospheres require an absolute accurate knowledge of the solar spectral irradiance $(S S I(\lambda))$. This is the case in the study of the Mars atmosphere with the Spectroscopy for Investigation of Characteristics of the Atmosphere of Mars (SPICAM) instrument (Montmessin et al., 2017). This instrument made resolved measurements of the planet radiance factor $(\operatorname{Rad}(\lambda)=B(\lambda) / S S I(\lambda))$ to derive key parameters (albedo, ozone, dust, etc.). In these studies, a decrease in the absolute level of the UV solar irradiance causes a decrease of ozone and dusts while the observed albedo of Mars increases. In addition, the irregularities in the UV spectrum like those observed in the SOLAR/SOLSTICE ratio (in the 216.9-226.8 nm spectral region), can have an impact on the radiance results.

To conclude, an improvement was made in the SOLAR-ISS spectrum (V2.0) to constrain the irregularity in the $216.9-226.8 \mathrm{~nm}$ range (Figure 2). Figure 3 (Right) shows the difference between the two versions of the SOLAR-ISS spectrum between $216.9 \mathrm{~nm}$ and $226.8 \mathrm{~nm}$.

\section{SOLAR-ISS improvements in the NIR}

As given in Equation 1, the SOLAR-ISS reference spectrum (V1.1) is obtained by combining the SOLAR/SOLSPEC measured spectrum (SOLAR-ISS (LR)) with reference solar spectra at high spectral resolution (Kurucz and Bell solar spectrum (1995) from 165 to $2400 \mathrm{~nm}$, and Solar Radiation Physical Modeling (SRPM) theoretical model (Fontenla, Stancil, and Landi, 2015) from 2400 to $3000 \mathrm{~nm})$.

To improve SOLAR-ISS (V1.1), it is necessary to compare it with high-resolution observations such as solar pseudo-transmissions. For comparisons with observed solar pseudo-transmissions, we remove the SOLAR-ISS (V1.1) data trend in the $2400-3000 \mathrm{~nm}$ spectral region using a low-order polynomial fit to obtain an equivalent SOLAR-ISS (V1.1) pseudo-transmission as shown in Figure 5 (Top). Figure 5 shows the solar pseudo-transmissions obtained with the Atmospheric Chemistry Experiment Fourier Transform Spectrometer (ACE-FTS) onboard a Canadian satellite designed to make observations of the Earth's atmosphere (SCISAT). The ACE-FTS instrument has a very high spectral resolution of $0.02 \mathrm{~cm}^{-1}$ and operates from 2.27 to $13.33 \mu \mathrm{m}\left(750-4400 \mathrm{~cm}^{-1}\right)$ using a Michelson interferometer (Chateauneuf et al., 2002; Soucy et al., 2002). Figure 5 also shows the Solar Pseudo-Transmittance Spectrum (SPTS) obtained by Toon (2017). The derived SPTS (with a solar disk-integrated spectrum) was computed using various recent versions of the solar line-list. SPTS (Toon, 2017) covers the $0.38-$ $16.67 \mu \mathrm{m}$ (600 to $26316 \mathrm{~cm}^{-1}$ ) spectral region.

Between 2400 and $3000 \mathrm{~nm}$, the comparisons between SOLAR-ISS (V1.1) and solar pseudo-transmissions (ACE-FTS and SPTS) show few abnormal solar lines in the SOLAR-ISS spectrum (V1.1) as shown in Figure 5 (Top). These solar features $(2431.3,2448.9,2469.9,2495.3,2526.1,2564.3,2612.7,2675.1,2758.3$, $2872.9 \mathrm{~nm})$ are too deep to be resolved with the SOLAR-ISS spectral resolution. Consequently, the SOLAR-ISS spectrum requires an improvement 
in the NIR using the high-resolution Kurucz and Bell (1995) data in the 2400$3000 \mathrm{~nm}$ spectral region.
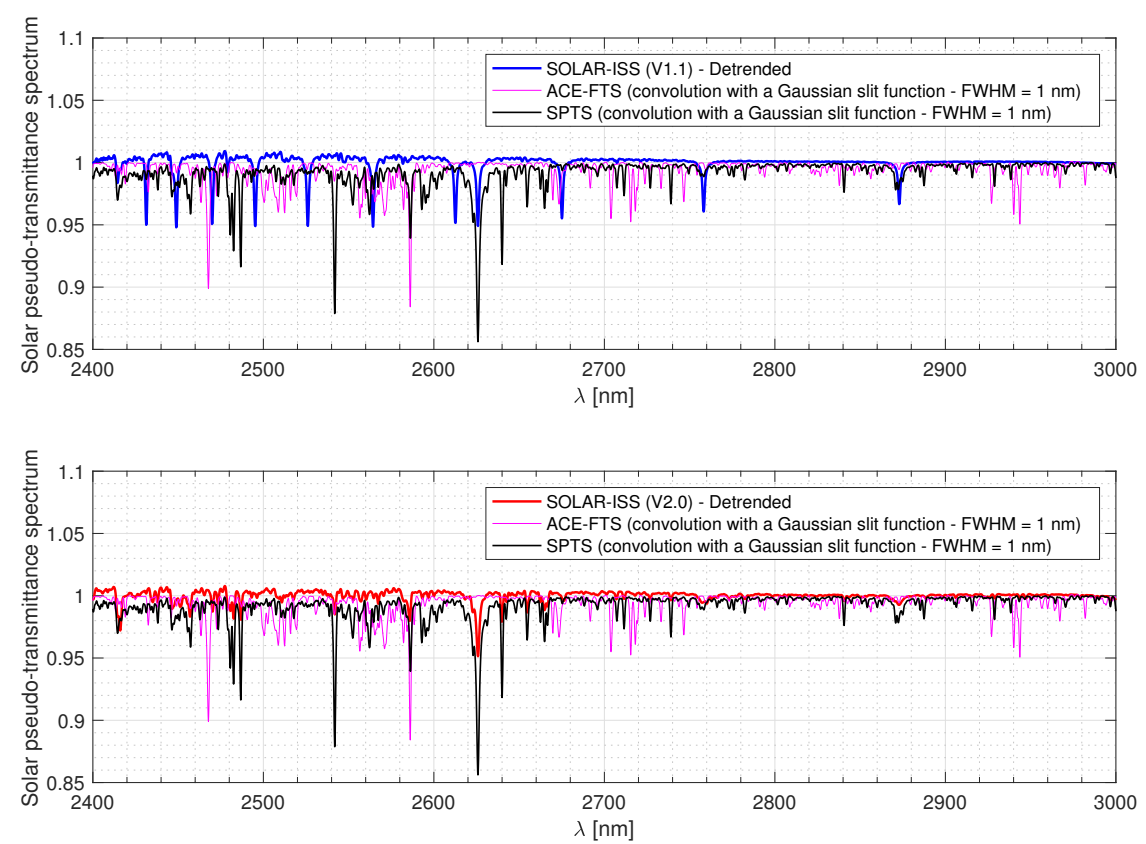

Figure 5. Top: Detrended SOLAR-ISS spectrum (V1.1) with a low-order polynomial fit and solar pseudo transmittances (ACE-FTS and SPTS) in the $2400-3000 \mathrm{~nm}$ spectral region. Bottom: Detrended SOLAR-ISS spectrum (V2.0) with a low-order polynomial fit and solar pseudo transmittances (ACE-FTS and SPTS) in the $2400-3000 \mathrm{~nm}$ spectral region.

\section{Results \& Discussion}

SOLAR/SOLSPEC observations cover almost the full solar cycle 24. We provided a traceable reference solar spectrum representative of the 2008 solar minimum (SOLAR-ISS - V1.1) using the SOLAR/SOLSPEC data thanks to revised engineering corrections, improved calibrations, and advanced procedures to account for thermal and aging corrections of the instrument. The new version of the SOLAR-ISS reference spectrum (V2.0) is presented in this section with all its improvements. A comparison between SOLAR-ISS (V2.0) and the new TSIS solar data is also made (Figure 6, Left), which represents a significant step in our quest for the determination of the 'true' solar spectrum. For this analysis, there is no adjustment between SOLAR-ISS (V2.0) and TSIS solar data, despite the fact that the comparison between these two datasets does not use coincident measurements.

For a given spectral region, other comparisons are made with other spectra from measurements (ATLAS 3, SORCE/SOLSTICE) and some models (Spectral And Total Irradiance REconstruction for the Satellite Era (SATIRE-S), Naval 

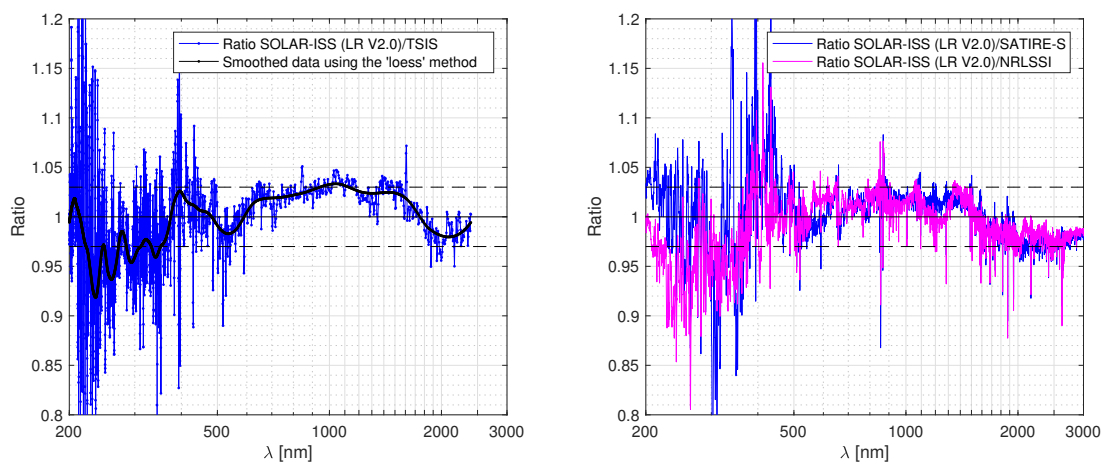

Figure 6. Left: Ratio to SOLAR-ISS (V2.0) for TSIS. Right: Ratio to SOLAR-ISS (V2.0) for SATIRE-S and NRL-SSI. We also observe that the correlations between the models (SATIRE-S and NRL-SSI) and TSIS are excellent.

Research Laboratory Solar Spectral Irradiance (NRL-SSI)). Coddington et al. (2018) paper provides, among other things, a comparison between more models (last version of the NRL-SSI2 modeled irradiance (Version 2), Solar Irradiance Data Exploitation (SOLID), SATIRE-S, a three-dimensional extension of the SATIRE-S model (SATIRE-3D), and Empirical Irradiance Reconstruction (EMPIRE)). In our case, we limit our comparisons with SATIRE-S and NRL-SSI (Figure 6, Right), which are well established models. We also did not focus on the revised solar spectral irradiance as measured by the Spectral Irradiance Monitor (SIM) instrument on board SORCE (Mauceri et al., 2018) because these results remain close to those proposed by models (SATIRE-S and NRL-SSI (V2)). SIM SSI (revised version) shows a good agreement with the SATIRE-S and NRL-SSI (V2) solar models within measurements uncertainties.

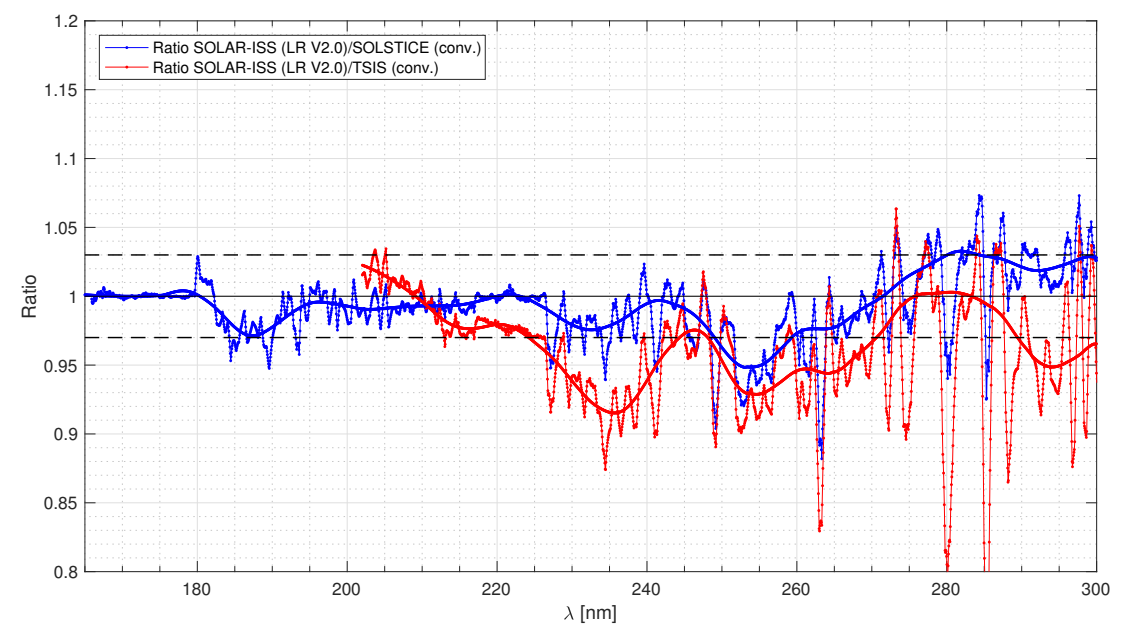

Figure 7. Ratio to SOLAR-ISS (V2.0) for SORCE/SOLSTICE (blue curve) and TSIS (red curve). These ratios are based on comparison between existing extraterrestrial solar spectra based on observations (SOLAR-ISS (April 2008), SORCE/SOLSTICE (April 2008), TSIS (March 2018 to March 2019)). The black dashed lines show the uncertainties at $\pm 3 \%$. 
Figure 7 shows a comparison between the SOLAR-ISS (V2.0) spectrum, the SORCE/SOLSTICE spectrum and the TSIS spectrum in the $165-300 \mathrm{~nm}$ spectral region. Differences appear between the three spectra. Figure 7 shows the good agreement between SOLAR-ISS data and SORCE/SOLSTICE data with a difference that, generally, does not exceed $\pm 3 \%$. However, there is broad spectral structure with width $\sim 20 \mathrm{~nm}$ between $220-300 \mathrm{~nm}$ in both the SOLARISS/SOLSTICE ratio (blue) and the SOLAR-ISS/TSIS ratio (red). This similarity could indicate wavelength-dependent issues at the $\sim 5 \%$ peak-to-peak level in the SOLAR-ISS calibration.

Between SOLAR-ISS and TSIS, differences are much larger. The solar spectral irradiance values measured by TSIS are generally higher than those proposed in SOLAR-ISS. We can not really conclude since these two spectra are obtained at different periods. These evolutions will nevertheless be the subject of a future discussion since these two spectra are associated with a solar minima. If the difference is real, this could imply that the solar minima are not at the same level involving a decrease or increase from one cycle to another one.

Although the SORCE/SOLSTICE and TSIS data both represent solar minimum conditions, it should be noted that the SORCE/SOLSTICE measurements occur $\sim 5$ years after the launch of SORCE, so the accuracy of the SORCE/SOLSTICE time-dependent calibration is a relevant factor. The quoted SORCE/SOLSTICE uncertainty of $0.5 \% /$ year would represent a $2.5 \%$ cumulative uncertainty at the time of these measurements. A recent paper by DeLand et al. (2019) shows that SORCE/SOLSTICE irradiances at $230-235 \mathrm{~nm}$ may be a few percent low by mid-2007 (their Figure 6). Increasing SOLSTICE irradiance values in this spectral region would lower the blue curve in Figure 7, and thus could bring the SOLAR-ISS/SOLSTICE and SOLAR-ISS/TSIS comparisons closer together. In the visible spectrum $(380-780 \mathrm{~nm})$ and in a part of the near infrared (780$2400 \mathrm{~nm}$ ), the solar spectral irradiance are identical in Versions 1.1 and 2.0 of the SOLAR-ISS reference spectrum. Between $380 \mathrm{~nm}$ and $2400 \mathrm{~nm}$, Figure 6 (Left) shows the excellent agreement between SOLAR-ISS and TSIS with a difference that does not exceed $\pm 3 \%$. Figure 6 (Right) shows a similar conclusion between SOLAR-ISS and the solar models (SATIRE-S and NRL-SSI).

The SOLAR-ISS spectrum is very consistent with TSIS and solar models in the $380-2400 \mathrm{~nm}$ spectral region. TSIS mostly confirms the SOLAR-ISS spectrum values above $1500 \mathrm{~nm}$. Figure 8 shows the brightness temperature of the Sun obtained for ATLAS 3, SOLAR-ISS (V2.0), and TSIS. Data of these three solar spectra are used to derive the brightness temperature of the Sun as a function of wavelength. This allows to better visualize the differences between the three solar spectra in the $380-2400 \mathrm{~nm}$ spectral region. Despite different periods of observation of these three solar spectra, it is possible to directly compare them because the solar variability in the $380-2400 \mathrm{~nm}$ spectral region is small during a solar cycle and much smaller than the uncertainties of the instruments. The main spectral region of disagreement between SOLAR-ISS (V2.0) and ATLAS 3 is clearly visible around $1640 \mathrm{~nm}$, where the solar atmosphere radiative opacity reaches its absolute minimum (in the photosphere at $\mathrm{T} \sim 6400 \mathrm{~K}$, the principal source of opacity is the $\mathrm{H}^{-}$ion where the free-bound process has its edge). Figure 8 clearly shows that the TSIS measurements follow those obtained by 
the SOLAR/SOLSPEC spectro-radiometer with very slight differences. TSIS observations are also consistent with all recent results (Elsey et al., 2017; Hilbig et al., 2018; Pereira et al., 2018).

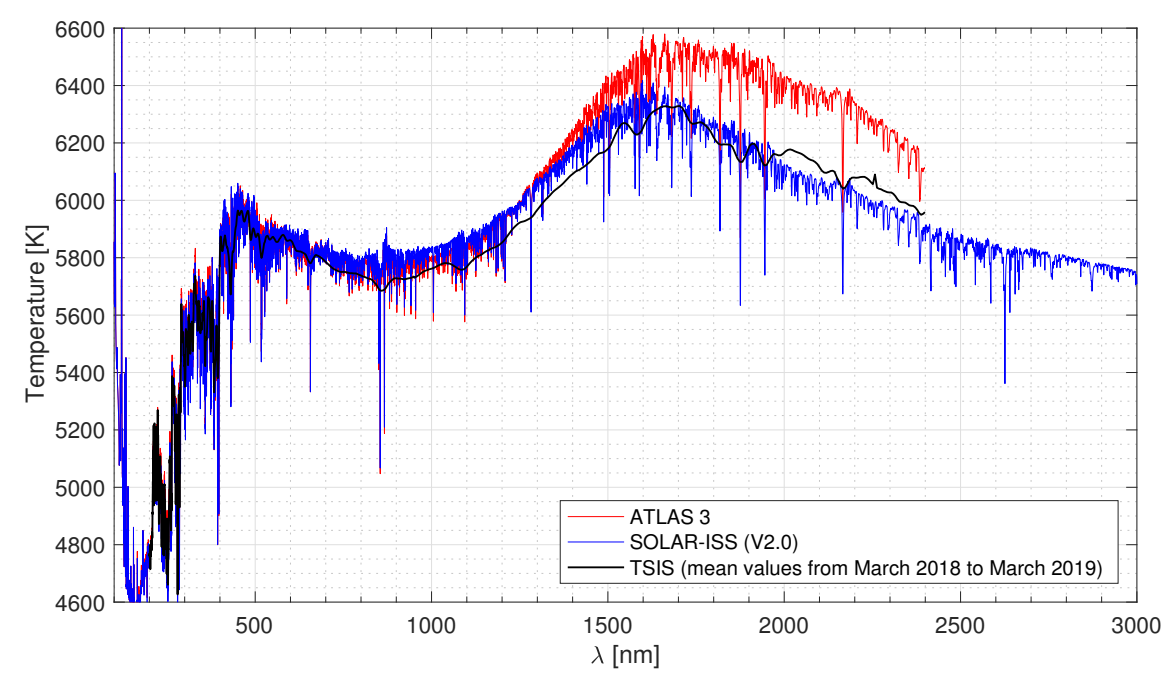

Figure 8. Brightness temperature of the Sun obtained from the ATLAS 3 (period: November 1994) solar spectrum data plot (red curve), SOLAR-ISS (period: April 2008) spectrum data (blue curve) and TSIS (period: March 2018 to March 2019) spectrum data (black curve).
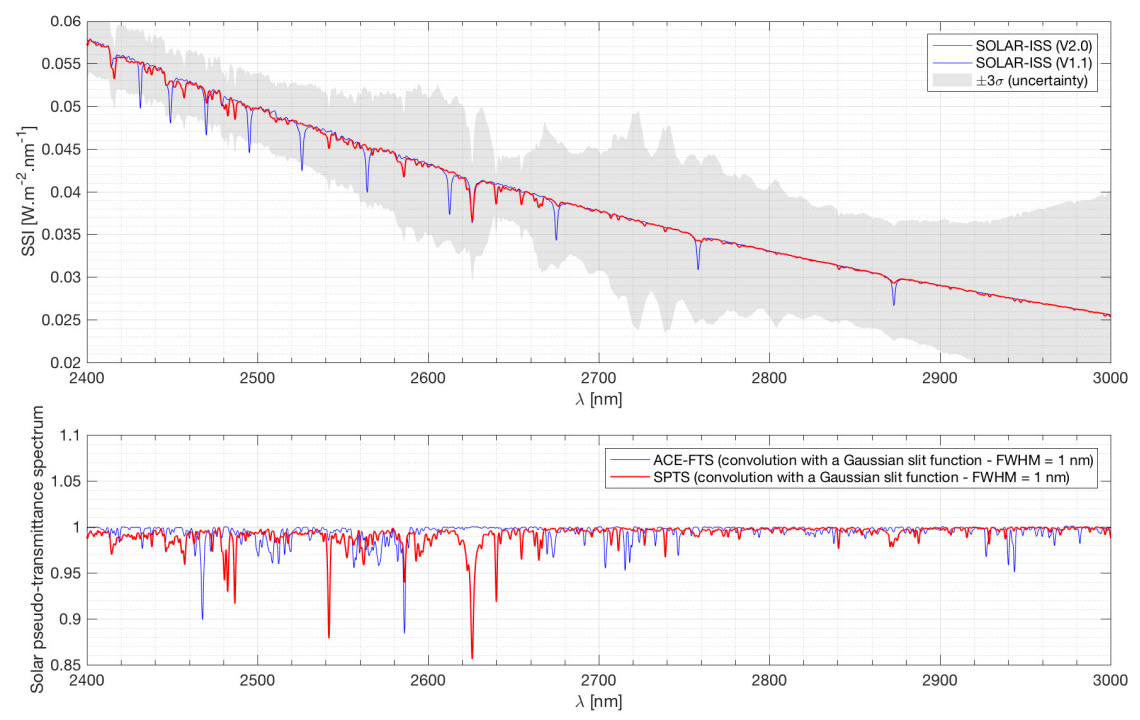

Figure 9. Top: Solar irradiance in the $2400-3000 \mathrm{~nm}$ spectral region of SOLAR-ISS (V2.0) shown in the red curve and SOLAR-ISS (V1.1) shown in the blue curve. The uncertainties are shown with the gray shaded curves. Bottom: Solar pseudo transmittance in the $2400-3000 \mathrm{~nm}$ spectral region of ACE-FTS and SPTS. The two sets of data are convolved with a Gaussian slit function, which has a full width at half maximum (FWHM) of $1 \mathrm{~nm}$. 
Figure 9 (Top) highlights the differences between the two versions of the SOLARISS spectrum (Section 4). In SOLAR-ISS (V1.1), above $2400 \mathrm{~nm}$, we used the SRPM theoretical model (with a resolution of $1 \mathrm{~nm}$ ) from Fontenla, Stancil, and Landi (2015). In SOLAR-ISS (V2.0), we fall back on the Kurucz and Bell (1995) studies. Then, the improvement in SOLAR-ISS (V2.0) between 2400-3000 nm is due to the use of the high-resolution Kurucz and Bell (1995) data.

The upgrade of the SOLAR-ISS spectrum above $2400 \mathrm{~nm}$ is related to the fact that we had deep abnormal solar lines in the version 1.1. Comparing with ACEFTS and SPTS solar pseudo transmittances in the $2400-3000 \mathrm{~nm}$ spectral region shown in Figure 9 (Bottom), we see that these solar lines (2431.3, 2448.9, 2469.9, 2495.3, 2526.1, 2564.3, 2612.7, 2675.1, 2758.3, 2872.9 nm) do not exist or are less deep. Between 2400 and $3000 \mathrm{~nm}$, SOLAR-ISS spectra have a resolution of $1 \mathrm{~nm}$. To be able to directly compare all data (SOLAR-ISS, ACE-FTS and SPTS), ACE-FTS and SPTS solar pseudo transmittances have been convolved with Gaussian slit functions, which allow to have the same resolution as the SOLAR-ISS spectrum.

Figure 10 presents the spectral dependence of the uncertainty for the SOLAR-ISS reference spectrum. These uncertainties are mainly derived from groundbased calibrations performed with the PTB blackbody (Meftah et al., 2018). They are also obtained from on-board diagnostics (flight calibrations) based on data temperature corrections (Meftah et al., 2017). Causes of the limitations are related to the fact that the radiometric responsivity of each SOLAR/SOLSPEC double-monochromator is considered as identical between pre-flight calibrations and first light in orbit.

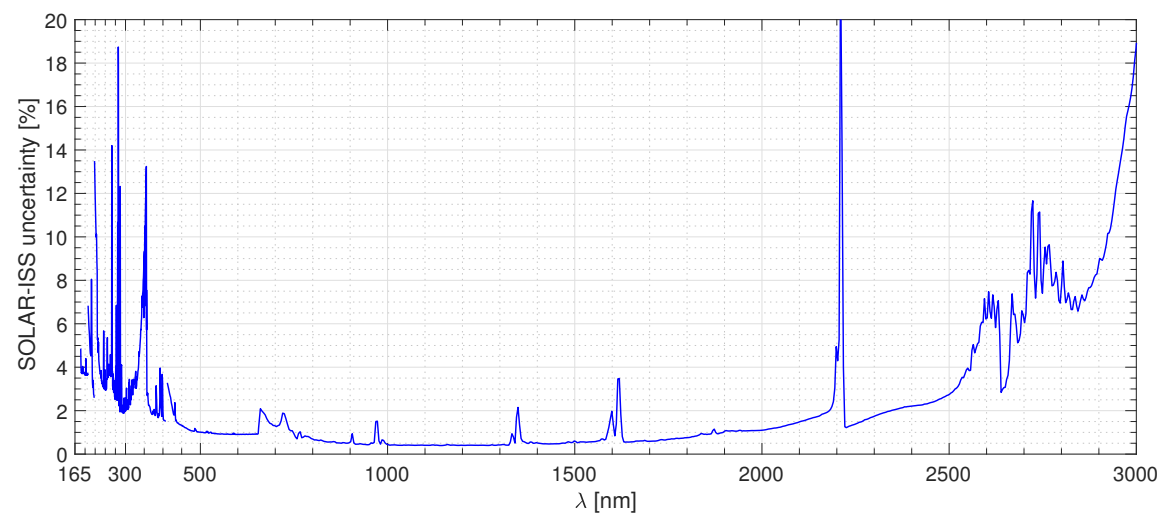

Figure 10. SOLAR-ISS uncertainties at $1 \sigma$ for all spectral regions.

\section{Conclusions}

SOLAR-ISS is the first solar spectrum obtained outside the atmosphere over a wavelength range of 165 to $3000 \mathrm{~nm}$ with a low level of uncertainty. The SOLARISS spectrum that is representative of the 2008 solar minimum challenged older 
solar reference spectra (ATLAS 3, WHI 2008), particularly in the near infrared above $1500 \mathrm{~nm}$. In this article, we propose a new version of the SOLAR-ISS spectrum (V2.0), where all the new corrections are described. SOLAR-ISS (V2.0) is compared with a new solar spectral irradiance dataset obtained thanks to the NASA TSIS-1 mission, which was deployed on the International Space Station in December 2017. First analysis between SOLAR-ISS and TSIS shows an excellent agreement between SOLAR-ISS and TSIS spectra with a difference that is not exceeding $\pm 3 \%$ in the $380-2400 \mathrm{~nm}$ spectral region. The TSIS measurements show an excellent agreement with the SOLAR-ISS spectrum for mostly all wavelengths above $1500 \mathrm{~nm}$. TSIS is consistent with the SOLAR-ISS spectrum such as all recent results of the SSI in the near infrared (CAVIAR 2, SCIAMACHY (V9), PYR-ILIOS).

The SOLAR-ISS (V2.0) spectrum is available at the Centre de Données astronomiques de Strasbourg (CDS).

Acknowledgements The SOLAR/SOLSPEC team acknowledges the support from European Space Agency (ESA), Centre National d'Études Spatiales (CNES, France), Centre National de la Recherche Scientifique (CNRS, France), the Programme National Soleil-Terre (PNST) from the Institut National des Sciences de l'Univers (INSU, France), the PROgramme de Développement d'Expériences scientifiques Office (PRODEX, Belgium), the Belgian Federal Science Policy Office (BELSPO) through the ESA-PRODEX program and LASP (USA). The LATMOS team gratefully acknowledges Kader Amsif (CNES), François Buisson (CNES), Denis Jouglet (CNES) and François Leblanc (CNRS) for their support in the implementation of a new solar reference spectrum. T. Hilbig, K. Bramstedt, and M. Weber acknowledge the support from Bundesministerium für Forschung und Technologie (Germany) via the SCIASOL project as part of the priority program ROMIC (Role of the Middle Atmosphere in Climate). The authors wish to thank the anonymous referees for the very useful comments, which improve the quality of the manuscript.

\section{References}

Bolsée, D.: 2012, Métrologie de la spectrophotométrie solaire absolue: principes, mise en oeuvre et résultats ; instrument solspec à bord de la station spatiale internationale (unpublished doctoral dissertation). PhD thesis, Université libre, École polytechnique de Bruxelles.

Bolsée, D., Pereira, N., Gillotay, D., Pandey, P., Cessateur, G., Foujols, T., Bekki, S., Hauchecorne, A., Meftah, M., Damé, L., Hersé, M., Michel, A., Jacobs, C., Sela, A.: 2017, SOLAR/SOLSPEC mission on ISS: In-flight performance for SSI measurements in the UV. Astron. Astrophys. 600, A21. doi:10.1051/0004-6361/201628234.

Brasseur, G.P., Solomon, S.: 2005, Aeronomy of the Middle Atmosphere: Chemistry and Physics of the Stratosphere and Mesosphere, 3rd.

Chateauneuf, F., Soucy, M.-A.A., Deutsch, C., Blanchard, N., Giroux, J.G.: 2002, Recent developments on the ACE-FTS instrument. In: Strojnik, M., Andresen, B.F. (eds.) Infrared Spaceborne Remote Sensing IX, Proc. SPIE 4486, 393-402. doi:10.1117/12.455122.

Coddington, O., Lean, J., Pilewskie, P., Snow, M.A., Kopp, G., Richard, E.C., Woods, T.N., DeLand, M.T., Marchenko, S.V.: 2018, A Comparative Assessment of Solar Irradiance Observations and Models at the Dawn of TSIS. AGU Fall Meeting Abstracts.

DeLand, M.T., Floyd, L.E., Marchenko, S., Tiruchirapalli, R.: 2019, Creation of the GSFCSSI2 Composite Solar Spectral Irradiance Data Set. Earth and Space Science 6(7), 1284-1298. doi:10.1029/2019EA000616. 
Elsey, J., Coleman, M.D., Gardiner, T., Shine, K.P.: 2017, Can Measurements of the NearInfrared Solar Spectral Irradiance be Reconciled? A New Ground-Based Assessment Between 4,000 and 10,000 $\mathrm{cm}^{-1}$. Geophys. Res. Lett. 44, 10. doi:10.1002/2017GL073902.

Fontenla, J.M., Landi, E.: 2018, Bright Network, UVA, and the Physical Modeling of Solar Spectral and Total Irradiance in Recent Solar Cycles. Astrophys. J. 861, 120. doi:10.3847/1538-4357/aac388.

Fontenla, J.M., Stancil, P.C., Landi, E.: 2015, Solar Spectral Irradiance, Solar Activity, and the Near-Ultra-Violet. Astrophys. J. 809, 157. doi:10.1088/0004-637X/809/2/157.

Gueymard, C.A.: 2018, Revised composite extraterrestrial spectrum based on recent solar irradiance observations. Solar Energy 169, 434-440. doi:10.1016/j.solener.2018.04.067.

Hilbig, T., Weber, M., Bramstedt, K., Noël, S., Burrows, J.P., Krijger, J.M., Snel, R., Meftah, M., Damé, L., Bekki, S., Bolsée, D., Pereira, N., Sluse, D.: 2018, The New SCIAMACHY Reference Solar Spectral Irradiance and Its Validation. Solar Phys. 293, 121. doi:10.1007/s11207-018-1339-9.

Kopp, G., Lean, J.L.: 2011, A new, lower value of total solar irradiance: Evidence and climate significance. Geophys. Res. Lett. 38, 1706. doi:10.1029/2010GL045777.

Kopp, G., Dudok de Wit, T., Ball, W.T., Finsterle, W., Frohlich, C., Kokkonen, K., Meftah, M., Schmutz, W.K.: 2018, The New "Community-Consensus TSI Composite" for Solar and Climate Researchers. AGU Fall Meeting Abstracts.

Kurucz, R.L., Bell, B.: 1995, Atomic line list.

Marchenko, S.V., DeLand, M.T., Lean, J.L.: 2016, Solar spectral irradiance variability in cycle 24: observations and models. J. Space Weather Space Clim. 6, A40. doi:10.1051/swsc/2016036. https://doi.org/10.1051/swsc/2016036.

Mauceri, S., Pilewskie, P., Richard, E., Coddington, O., Harder, J., Woods, T.: 2018, Revision of the Sun's Spectral Irradiance as Measured by SORCE SIM. Solar Phys. 293, 161. doi:10.1007/s11207-018-1379-1.

McClintock, W.E., Rottman, G.J., Woods, T.N.: 2005, Solar-Stellar Irradiance Comparison Experiment II (Solstice II): Instrument Concept and Design. Solar Phys. 230, 225-258. doi:10.1007/s11207-005-7432-x.

Meftah, M., Dewitte, S., Irbah, A., Chevalier, A., Conscience, C., Crommelynck, D., Janssen, E., Mekaoui, S.: 2014, SOVAP/ Picard, a Spaceborne Radiometer to Measure the Total Solar Irradiance. Solar Phys. 289, 1885-1899. doi:10.1007/s11207-013-0443-0.

Meftah, M., Bolsée, D., Damé, L., Hauchecorne, A., Pereira, N., Irbah, A., Bekki, S., Cessateur, G., Foujols, T., Thiéblemont, R.: 2016, Solar Irradiance from 165 to $400 \mathrm{~nm}$ in 2008 and UV Variations in Three Spectral Bands During Solar Cycle 24. Solar Phys. 291, 3527-3547. doi:10.1007/s11207-016-0997-8.

Meftah, M., Damé, L., Bolsée, D., Pereira, N., Sluse, D., Cessateur, G., Irbah, A., Sarkissian, A., Djafer, D., Hauchecorne, A., Bekki, S.: 2017, A New Solar Spectrum from 656 to 3088 nm. Solar Phys. 292(8), 101. doi:10.1007/s11207-017-1115-2.

Meftah, M., Damé, L., Bolsée, D., Hauchecorne, A., Pereira, N., Sluse, D., Cessateur, G., Irbah, A., Bureau, J., Weber, M., Bramstedt, K., Hilbig, T., Thiéblemont, R., Marchand, M., Lefèvre, F., Sarkissian, A., Bekki, S.: 2018, SOLAR-ISS: A new reference spectrum based on SOLAR/SOLSPEC observations. Astron. Astrophys. 611, A1. doi:10.1051/0004$6361 / 201731316$

Menang, K.P.: 2018, Assessment of the Impact of Solar Spectral Irradiance on Near-Infrared Clear-Sky Atmospheric Absorption and Heating Rates. Journal of Geophysical Research (Atmospheres) 123, 6460-6468. doi:10.1029/2018JD028342.

Montmessin, F., Korablev, O., Lefèvre, F., Bertaux, J.-L., Fedorova, A., Trokhimovskiy, A., Chaufray, J.Y., Lacombe, G., Reberac, A., Maltagliati, L., Willame, Y., Guslyakova, S., Gérard, J.-C., Stiepen, A., Fussen, D., Mateshvili, N., Määttänen, A., Forget, F., Witasse, O., Leblanc, F., Vandaele, A.C., Marcq, E., Sandel, B., Gondet, B., Schneider, N., Chaffin, M., Chapron, N.: 2017, SPICAM on Mars Express: A 10 year in-depth survey of the Martian atmosphere. Icarus Journal 297, 195-216. doi:10.1016/j.icarus.2017.06.022.

Pereira, N., Bolsée, D., Sperfeld, P., Pape, S., Sluse, D., Cessateur, G.: 2018, Metrology of solar spectral irradiance at the top of the atmosphere in the near infrared measured at Mauna Loa Observatory: the PYR-ILIOS campaign. Atmospheric Measurement Techniques 11, 6605 - 6615. doi:10.5194/amt-11-6605-2018.

Richard, E.C., Pilewskie, P., Kopp, G., Coddington, O., Woods, T.N., Wu, D.L.: 2016, Continuing the Solar Irradiance Data Record with TSIS. AGU Fall Meeting Abstracts.

Richard, E.C., Harber, D., Coddington, O., Beland, S., Chambliss, M., Mauceri, S., Pilewskie, P.: 2018, Implementation of Solar Spectral Irradiance Measurements from the International 
Space Station: The TSIS-1 First Light and Early Mission Results. AGU Fall Meeting Abstracts.

Rottman, G.: 2005, The SORCE Mission. Solar Phys. 230, 7-25. doi:10.1007/s11207-0058112-6.

Schmutz, W., Fehlmann, A., Finsterle, W., Kopp, G., Thuillier, G.: 2013, Total solar irradiance measurements with PREMOS/PICARD. In: American Institute of Physics Conference Series, American Institute of Physics Conference Series 1531, 624-627. doi:10.1063/1.4804847.

Shapiro, A.I., Schmutz, W., Schoell, M., Haberreiter, M., Rozanov, E.: 2010, NLTE solar irradiance modeling with the COSI code. Astron. Astrophys. 517, A48. doi:10.1051/0004$6361 / 200913987$.

Snow, M., McClintock, W.E., Woods, T.N.: 2010, Solar spectral irradiance variability in the ultraviolet from sorce and uars solstice. Advances in Space Research 46(3), $296 \quad-\quad 302$. doi:https://doi.org/10.1016/j.asr.2010.03.027. http://www.sciencedirect.com/science/article/pii/S0273117710002322.

Snow, M., Eparvier, F.G., Harder, J., Jones, A.R., McClintock, W.E., Richard, E., Woods, T.N.: 2018, Ultraviolet Solar Spectral Irradiance Variation on Solar Cycle Timescales. In: Banerjee, D., Jiang, J., Kusano, K., Solanki, S. (eds.) IAU Symposium, IAU Symposium 340, 203-208. doi:10.1017/S1743921318001278.

Soucy, M.-A.A., Chateauneuf, F., Deutsch, C., Etienne, N.: 2002, ACE-FTS instrument detailed design. In: Barnes, W.L. (ed.) Earth Observing Systems VII, Proc. SPIE 4814, 70 - 81. doi: $10.1117 / 12.451701$

Tagirov, R.V., Shapiro, A.I., Schmutz, W.: 2017, NESSY: NLTE spectral synthesis code for solar and stellar atmospheres. Astron. Astrophys. 603, A27. doi:10.1051/0004$6361 / 201628574$

Thuillier, G., Hersé, M., Labs, D., Foujols, T., Peetermans, W., Gillotay, D., Simon, P.C., Mandel, H.: 2003, The Solar Spectral Irradiance from 200 to $2400 \mathrm{~nm}$ as Measured by the SOLSPEC Spectrometer from the Atlas and Eureca Missions. Solar Phys. 214, 1. doi:10.1023/A:1024048429145.

Thuillier, G., Foujols, T., Bolsée, D., Gillotay, D., Hersé, M., Peetermans, W., Decuyper, W., Mandel, H., Sperfeld, P., Pape, S., Taubert, D.R., Hartmann, J.: 2009, SOLAR/SOLSPEC: Scientific Objectives, Instrument Performance and Its Absolute Calibration Using a Blackbody as Primary Standard Source. Solar Phys. 257, 185-213. doi:10.1007/s11207009-9361-6.

Toon, G.C.: 2017, Solar line list for the tccon 2014 data release, CaltechDATA. doi:10.14291/tccon.ggg2014.solar.r0/1221658. https://data.caltech.edu/records/251.

Woods, T.N., Chamberlin, P.C., Harder, J.W., Hock, R.A., Snow, M., Eparvier, F.G., Fontenla, J., McClintock, W.E., Richard, E.C.: 2009, Solar Irradiance Reference Spectra (SIRS) for the 2008 Whole Heliosphere Interval (WHI). Geophys. Res. Lett. 36, L01101. doi:10.1029/2008GL036373. 